\title{
Numerical Prediction of Non-Cavitating and Cavitating Vortex Rope in a Francis Turbine Draft Tube
}

\author{
Dragica Jošt* - Andrej Lipej \\ Turboinštitut, Ljubljana, Slovenia
}

The paper presents a prediction of vortex rope in a draft tube obtained by the numerical flow analysis. The main goal of the research was to numerically predict pressure pulsation amplitude versus different guide vanes openings and compare the results with experimental ones. Three turbulent models (SAS-SST, $\omega$-RSM and LES) were used. Also the effect of different domain configurations, grid density and time step size on results was examined. At first analysis was done without cavitation, while later at one operating point the cavitation model was included.

(C)2011 Journal of Mechanical Engineering. All rights reserved.

Keywords: Francis turbine, vortex rope, cavitation, pressure pulsation, turbulent models

\section{INTRODUCTION}

Pressure fluctuations are a serious problem in hydraulic machinery and are usually the result of a strong vortex created in a centre of a flow at the outlet of a runner. The draft tube vortex appears at partial load operating regimes usually in radial turbines and also at single regulated axial turbines. The consequences of the vortex developed in the draft tube are very unpleasant pressure pulsation, axial and radial forces and torque fluctuation as well as turbine structure vibration.

The intensive investigations of draft tube pressure pulsation on various specific speed turbines were performed in 1990's and the results are published in [1] to [3]. Tests were performed in the wide turbine operating range from partial to full load and from runaway to head above optimum. Pressure pulsations were measured on models and prototypes. The influence of cavitation and various kinds of air admission on the pressure pulsation as well as on the efficiency characteristics were investigated. The conclusion was that the intensity of the vortex depends on specific speed of the turbine, operating regime and especially on a shape of runner blades and channel. Significantly different dynamic characteristics can be obtained with different runner shapes at the same specific speed and approximately the same energetic characteristics. Unfortunately, the results from the model test are not always valid for the prototype and an acceptable model can be an unpleasant surprise as a prototype. Therefore, an accurate numerical prediction of the vortex existence and intensity in a design stage is an important task.

The first attempt to numerically simulate the unsteady flow pattern accompanied with the helical vortex was made by Skotak [4] and [5]. Large Eddy Simulation (LES) model was used and in spite of for this model very coarse grid he managed to get a low pressure zone, which agrees well with the rotating rope observed in experiment. In the following years several papers about this topic were published. Usually unsteady analysis was performed only in the draft tube and the results of the previous steady state analysis of the runner were used as inlet boundary conditions. In some cases the runner and the draft tube were analyzed simultaneously. Most of the authors reported that by standard $k-\varepsilon$ model no rotating rope was obtained, while by the extended $k-\varepsilon$ model of Kim and Chen [6] and realizable $k-\varepsilon$ model [7] the rotating rope was obtained, but it was overly damped. Better results were obtained by Reynolds Stress Models (RSM) [7] and [8] and Large Eddy Simulation (LES) [9] and [10]. Generally, where the experimental results were available, the frequency of pressure pulsation matched the measured values quite well, but the prediction of amplitudes was less accurate. When the cavitation was included [9], [11] and [12], it was reported that numerically obtained cavitating rope was smaller than in the experimental observation while the frequencies and amplitudes of pressure pulsation were not compared with

*Corr. Author's Address: Turboinštitut, 
the experimental values. The frequencies were the same whether cavitation was modeled or not, but amplitudes were smaller in case of cavitation modeling. Due to long computational time, in most papers mentioned above, unsteady numerical analysis was limited to one or two operating points, computational grids were rather coarse and in some cases more time steps would be needed to get reliable results. In addition to the numerical flow analysis, an interesting approach to better understand these phenomena is simultaneous flow visualization and measurements of structural fluctuations [13].

This paper is a continuation of the work presented in [14] and [15]. In [14] the results obtained by the SAS-SST model at four operating points were presented. In [15] a numerical simulation at one operating point was performed by three turbulent models (SAS-SST, RSM and LES), with and without cavitation. Due to very long computational time work was not completed and only preliminary results were presented. In this paper the results of [14] and [15] are summarized and completed.

\section{FLOW DISTRIBUTION AT THE RUNNER OUTLET AT PART LOAD}

For part load conditions, the vortex (also called rope) spirals outward and processes in the direction of the turbine's rotation typically between one fourth and one third of the turbine's rotational frequency. These phenomena can cause large pressure fluctuations, low frequency vibrations and undesirable variations in the turbine output.

At part load a turbine works with a relative guide vanes opening, which is smaller than at optimal operating regime. The runner channel is not uniformly fulfilled with the flow because the main flow is near the shroud. A great secondary backflow zone is formed near the hub (Fig. 1). On the border between backflow and mainstream there is a strong tangential shear, which is the main reason for the vortices.

Due to the the direction of circumferential component of absolute velocity the vortex has the same direction as the runner and goes downstream towards the draft tube. The vortex has a spiral shape and depending on the outlet velocity conditions it can have one, two or even three branches. Inside the strong vortex the pressure is very low and if it reaches the value of vapour pressure the cavitation is also present.

The shape of the runner channel and the distribution of circumferential and meridional velocity components, which depends on the runner blade shape, have an important influence on the distribution of the vorticity. At some runners the vortex is very weak also at extreme part load regime, but in some cases the vortex can be very strong with high amplitudes of the pressure pulsations. Unfortunately, it is not possible to predict the direct connection between the runner shape and dynamic characteristics of the turbine. It is for this reason necessary to complete the research work in this area, especially numerical prediction of the vortex formation and pressure pulsations characteristics, before any part of the turbine is constructed.

\section{NUMERICAL MODELING}

Flow in water turbines is turbulent and unsteady. While the efficiency and cavitation in Francis, Kaplan and bulb turbines can be predicted by a steady state flow analysis and the results are usually accurate enough, unsteady flow analysis has to be performed when unsteady phenomena such as rotating vortex rope are the objects of interest. In these cases also more advanced turbulent models as Reynolds Stress Models (RSM), Large Eddy Simulation (LES), Detached Eddy Simulation (DES) or Scale-Adaptive Simulation (SAS) models have to be used. In this paper three turbulent models are used: SAS-SST, RSM and LES.

The Scale-Adaptive Simulation (SAS) is an improved URANS (Unsteady Reynolds Averaged Navier-Stokes) formulation, which allows the resolution of the turbulent spectrum in unstable flow conditions. The SAS concept is based on the introduction of the von Karman length-scale into the turbulence scale equation. The information provided by the von Karman length-scale allows SAS models to dynamically adjust to resolved structures in a URANS simulation, which results in a LES-like behaviour in unsteady regions of the flow-field. At the same time, the model provides standard RANS capabilities in stable flow regions. SAS-SST model is the combination of SAS and SST (Shear Stress Transport) model. 
Reynolds Stress Turbulence Models (RSM) based on transport equations for all components of the Reynolds stress tensor and the dissipation rate. The exact production term and the inherent modelling of stress anisotropies make RSM more suited to complex flows, for example a flow with a rotating vortex rope in the draft tube, where standard two-equation models fail. However, due to six additional transport equations the computational time increases significantly.

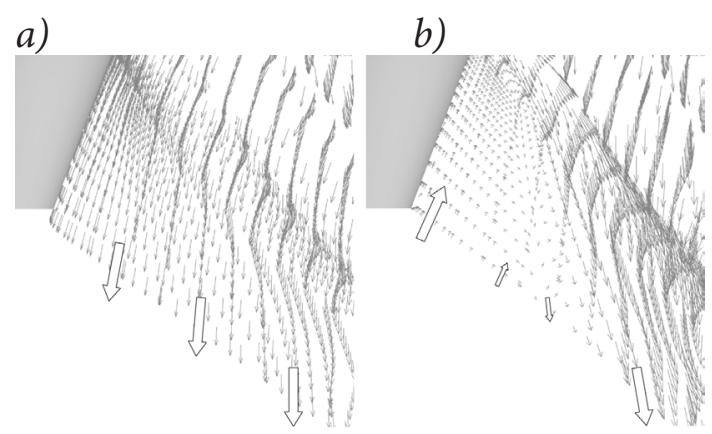

Fig. 1. Velocity distribution at the outlet of the runner for different operating regimes; a) $B E P, b$ ) part load

Details on the structure of turbulent flows, such as pressure fluctuations, can be obtained by LES. LES is an approach which solves for large-scale fluctuating motions and uses "subgrid « scale turbulence models for the smallscale motion. In ANSYS three LES models are available: the Smagorinsky model, the walladapted local eddy-viscosity model (LES WALE) and the Dynamic Smagorinsky-Lilly model. The first two models are algebraic. The Smagorinsky model is available together with two different formulations of the wall damping function. The LES WALE model needs no wall damping, while the Dynamic Smagorinsky-Lilly model uses the information contained in the resolved turbulent velocity field to evaluate the model coefficient. The method needs explicit (secondary) filtering and it is therefore more time consuming than the algebraic models. LES requires fine grids and small time steps, particularly for wall bounded flows, as well as a large number of time steps to generate statistically meaningful correlations for the fluctuation velocity components [16].

The cavitating vortex rope has to be modeled by one of the multiphase models. The homogeneous and inhomogeneous multiphase models are available. In the inhomogeneous model each fluid possesses its own field and the fluids interact via interphase transfer terms. There is one solution field for each separate phase. Sub-models differ in the way they model the interfacial area density and the interphase terms. The homogeneous model assumes that transported quantities (with the exception of volume fraction) for the process are the same for all phases. Therefore, it is sufficient to solve bulk transport equations for shared fields rather than solving individual transport equations. Density and viscosity are calculated from density and viscosity of all phases in the fluid. The cavitation is usually modeled by homogeneous model.

Cavitation refers to the process by which vapour forms in low pressure regions of a liquid flow. In the ANSYS CFX-12, The RayleighPlesset model is implemented in the multiphase framework as an interphase mass transfer model. The growth of bubbles is given by RayleighPlesset equation. The equation for volume fraction is fully coupled with flow equations, because the term, which represents the sources, depends on the pressure. The bubble grows if the pressure is low. On the other hand, the vaporization causes change of density in flow equations.

A detailed description of turbulent models and multiphase models can be found in ANSYS CFX-12 Solver Theory Documentation [16].

\section{MODEL TEST}

Pressure fluctuations on Francis model turbine at Turboinštitut are observed and measured in accordance with IEC 90193. For this purpose KISTLER piezoresistive absolute pressure transducers are located on different locations at the spiral casing and at the inlet part of the draft tube. The main purpose of these measurements was to obtain enough information about the magnitude and the nature of pressure fluctuations, their dominant frequency and additionally the dampening effect of air admission.

Signals from the pressure transducers are wired to the multi channel data acquisition system, based on National Instruments multifunction card with additional SCXI signal conditioning modules. Signals are acquired continuously with 
$5 \mathrm{kHz}$ sampling frequency and 16 bit resolution. Binary data samples stored on the computer hard disk are at least 30 seconds long. LabVIEW software is used to record and analyze pressure signals.

\section{NUMERICAL PREDICTION OF VORTEX ROPE}

Accuracy of numerical prediction of vortex rope was verified on two Francis turbines. For the first one (case 1) numerical analysis was performed at four operating points by SAS-SST turbulent model on different computational grids and domain configurations and with different time steps. Analysis was done without cavitation. The second turbine (case 2) was analyzed at one operating point by RSM, SAS-SST and LES turbulent models. To obtain the cavitating vortex rope homogenious two-phase flow model was used.

\subsection{Test Case 1: Prediction of Pressure Pulsation Amplitudes and Frequencies for Different Operating Regimes}

For the first turbine different computational grids and domain configurations were used (Table 1). At first, the computational grid of the complete turbine including spiral casing with stay vanes, guide vane cascade, runner and draft tube with 3,300,000 elements was used. The second computational grid of the complete turbine consists of the total $17,000,000$ elements. The third configuration consists of the runner and the draft tube with 25,000,000 elements. The grid of the spiral casing is made using tetrahedral elements with prism layers near the walls, all other turbine parts are meshed by hexahedral elements. The length of the time step in unsteady calculation was equal to one, three or six degrees of the runner revolution. Convergence criteria was prescribed to $5 \times 10^{-5}$ for RMS and in the average a converged solution was obtained after three to ten iterations in each time step, depending on the flow rate operating regime. Shape of the vortex rope at OP1, OP2 and OP3 is presented as isosurface of constant pressure (Fig. 2). Its exact value is in this case insignificant as cavitation was not modeled and the value of reference pressure value has no influence on the results. At OP4 no vortex rope was observed and pressure pulsations were negligible.

The numerically obtained frequency of the vortex rope is about 20 to $26 \%$ of the runner rotational speed depending on the operating regime. The frequency spectrum obtained from the measurements on the model test rig is very similar to the numerically obtained values. The difference between numerical and experimental results is about $1 \%$ of the runner rotational speed. In Table 3 the values of pressure pulsation frequency obtained by measurement and numerical analysis are presented.

The effect of grid density and time step can be seen in Fig. 3 where pressure pulsations at OP2 for two grid configurations are presented. For the coarse grid the time step was equal to 3 deg. of runner revolution. For the fine grid with 17 million elements the first part of the graph shows the pressure pulsation for time step equal to $6 \mathrm{deg}$. and the last part for time step equal to $1 \mathrm{deg}$. of runner revolution. In this case, the differences in frequency and amplitude due to the time step are negligible, but the effect of grid density can be clearly seen. The amplitudes of pressure pulsation calculated on the fine grid are significantly higher than those obtained on the coarse grid.

The calculation took a considerable amount of time since during the calculation the shape of the vortex rope was forming and only after several vortex revolutions the correct frequency was obtained. In Fig. 4 the history of the frequency during the calculation is shown for operating points OP1 and OP2. At the beginning of the calculation the vortex rope frequency was lower than the frequency obtained by the measurements. After five complete vortex revolutions the numerical and experimental values of the frequency differ for about one percent.

In addition to the vortex rope frequency the main challenge of the numerical analysis was the prediction of the pressure pulsation amplitudes for different operating regimes. The comparison between the measurements and the numerical results shows that the numerical prediction of the position of maximal pressure pulsation amplitude is quite accurate, but the predicted values of the amplitudes are lower than the experimental ones, except for the operating regime with the 
Table 1. Number of elements in particular geometry configuration, case 1

\begin{tabular}{|c|c|c|c|c|}
\hline & $\begin{array}{c}\text { Spiral casing, stay } \\
\text { and guide vanes }\end{array}$ & Runner & Draft tube & Total \\
\hline Configuration 1 & $1,400,000$ & $1,000,000$ & 900,000 & $3,300,000$ \\
\hline Configuration 2 & $1,400,000$ & $12,600,000$ & $3,000,000$ & $17,000,000$ \\
\hline Configuration 3 & - & $12,600,000$ & $12,400,000$ & $25,000,000$ \\
\hline
\end{tabular}

a)

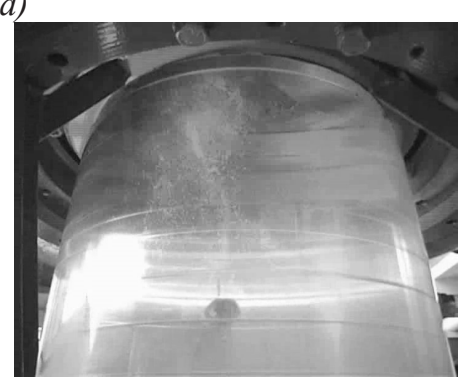

d)

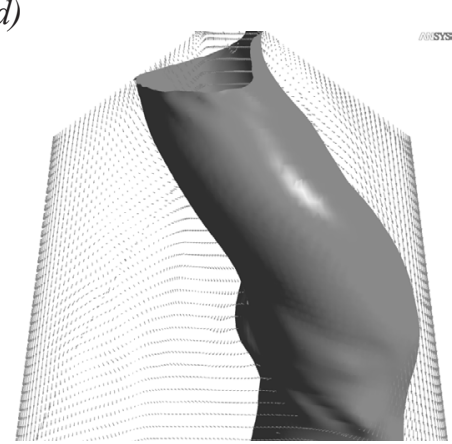

b)

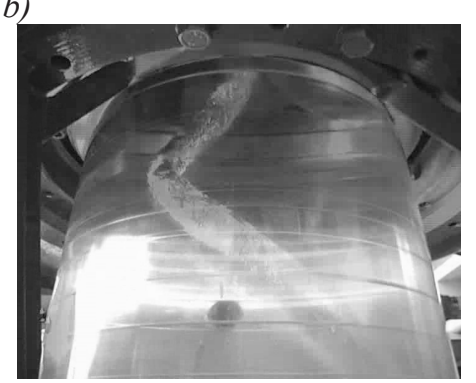

e)

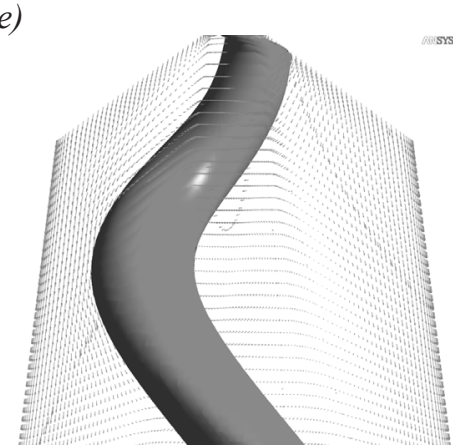

c)

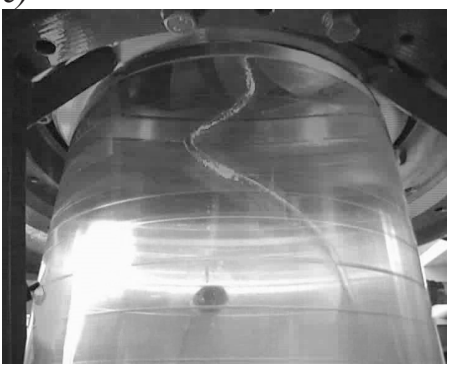

f)

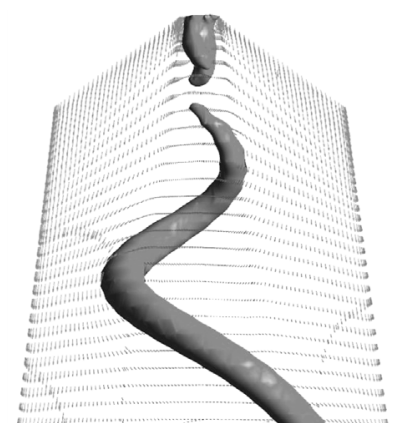

Fig. 2. Vortex rope at different operating points; a) experiment $O P 1, b)$ experiment $O P 2$, c) experiment $O P 3$, d) numerical simulation $O P 1$, e) numerical simulation $O P 2, f)$ numerical simulation OP3; case 1

smallest flow rate. A significant difference is obtained in pressure pulsation amplitudes for two different computational grids. The detailed results and comparison with measurements are presented in Fig. 5. It can be seen that with coarse computational grid (configuration 1) the difference between the numerical and experimental results

Table 2. Operating points for numerical analysis, case 1

\begin{tabular}{|c|c|c|c|}
\hline $\begin{array}{c}\text { Operating } \\
\text { point }\end{array}$ & $A_{0} / A_{0 \mathrm{BEP}}$ & $\varphi / \varphi_{\mathrm{BEP}}$ & $\psi / \psi_{\mathrm{BEP}}$ \\
\hline OP1 & 0.656 & 0.66 & 0.97 \\
\hline OP2 & 0.800 & 0.81 & 0.97 \\
\hline OP3 & 0.840 & 0.85 & 0.97 \\
\hline OP4 & 1.000 & 1.00 & 0.97 \\
\hline
\end{tabular}

at OP2 is about $44 \%$, but with grid refinement (configuration 2) the accuracy improved and the difference is less than $14 \%$.

\subsection{Test Case 2: The Effect of Turbulent Models}

In case 2 we focused on the effect of different turbulent models on the results. Analysis was done by SAS-SST, RSM and LES with and without cavitation. Cavitation was modeled by homogenious two-phase model. Mass transfer was done by the Rayleigh-Plesset model. In all the cases steady state solution without cavitation was used as an initial for steady state calculation with cavitation and these results were used as an initial for unsteady analysis with cavitation. 
Table 3. Pressure pulsation frequency - experimental and numerical results, case 1

\begin{tabular}{|c|c|c|c|c|}
\hline \multirow{2}{*}{ Operating point } & Experimental & \multicolumn{3}{|c|}{ Numerical values [Hz] } \\
\cline { 3 - 5 } & values [Hz] & configuration 1 & configuration 2 & configuration 3 \\
\hline OP1 & 3.20 & 3.09 & 3.22 & - \\
\hline OP2 & 4.00 & 4.01 & 3.95 & 3.80 \\
\hline OP3 & 4.10 & 3.80 & 3.82 & - \\
\hline OP4 & 4.45 & - & - & - \\
\hline
\end{tabular}

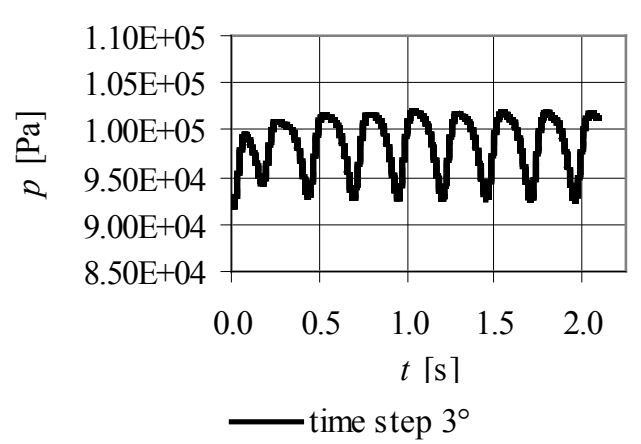

a)

Fig. 3. Pressure pulsations at OP2 for two computational grids; a) 3.3 million elements , b) 17 million elements, case 1

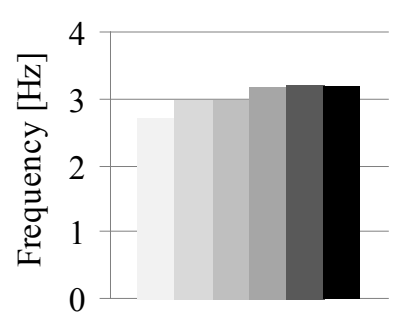

a)

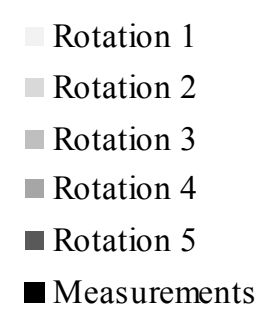

Measurements

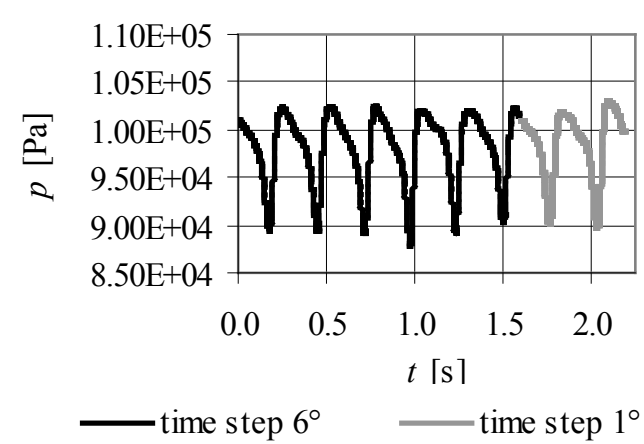

)

Fig. 4. Vortex rope frequency variations during the calculation at; a) OP1, b) OP2 compared to experimental values, case 1

Analysis was done for an operating point at part load $\left(A_{0} / A_{o B E P}=0.8572, \varphi / \varphi_{B E P}=0.7716\right.$, $\left.\psi / \psi_{B E P}=0.7850\right)$, for which pressure was measured at two positions on the conical part of the draft tube (Fig. 6) and quite strong pressure pulsations were detected. The value of cavitation coefficient $\sigma$ was equal to 0.1497 . For this value of $\sigma$ the cavitation was present and due to the water vapour in the rope its long thick shape was seen (Fig. 7).

When cavitation is modeled the value of cavitation coefficient in numerical analysis has to be the same as in reality. Therefore, the value of static pressure prescribed at the outlet of the draft tube was obtained from the measurements.

The domain of calculation, when SAS-SST and RSM were used, was the complete turbine. Computational grid consists of 5.6 million nodes and is rather coarse, only the grid in the draft tube was a bit refined and it consists of 3.4 million nodes. Unsteady analysis by $\omega$-RSM model started from the steady state solution obtained by the same turbulent model, while the analysis with SAS-SST model started from the steady state solution obtained by the SST model.

Time step was equal to 2 degrees of runner revolution. When the cavitation model was 


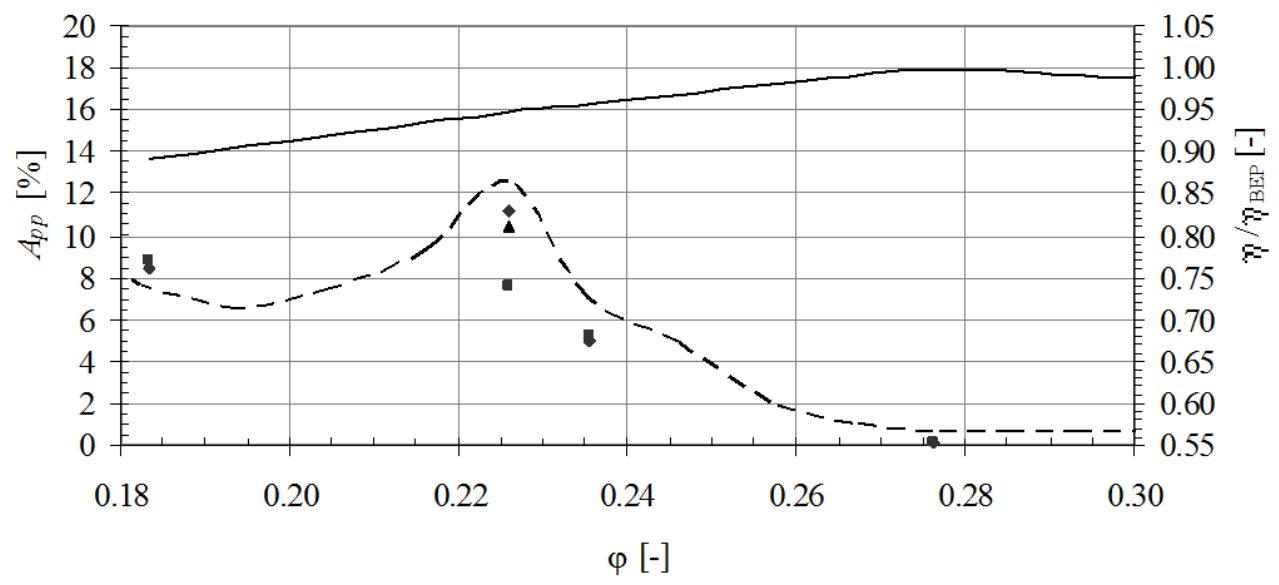
---- experiment
- CFD, configuration 1
- CFD, configuration 2
- CFD, configuration 3
CFD, efficiency

Fig. 5. Pressure pulsation, $98 \%$ peak-to-peak amplitudes for different operating regimes; comparison between experimental and numerical values, case 1

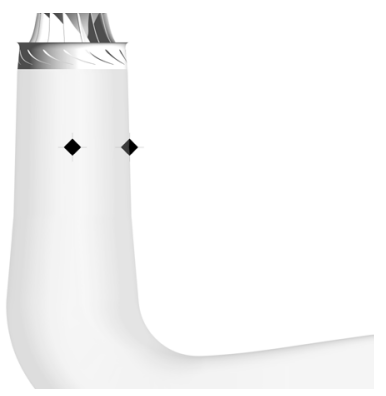

Fig. 6. The positions of pressure pulsation measurement, case 2

included the $\omega$-RSM model was replaced by BSLRSM and the time step was reduced to 1 degree of runner revolution.

LES requires fine grid, therefore the domain of the calculation was reduced to the draft tube with prolongation at the outlet. The grid consists of 23.5 million nodes. At the inlet of the draft tube velocity components obtained by an analysis of the whole turbine by SST turbulent model were prescribed. The results of the steady state solution obtained by the SST model were used as an initial condition. The runner rotation was simulated by unsteady inlet condition. Time step corresponded to $0.5 \mathrm{deg}$. of runner revolution.

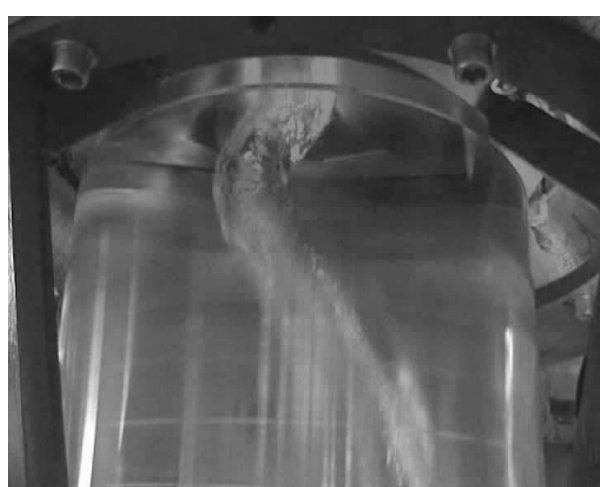

Fig. 7. Cavitating vortex rope on test rig, case 2

The average Courant number was around 0.2. For each time step 4 to 6 iterations were needed. Calculation without cavitation was done by the Smagorinsky model. When analysis was repeated with cavitation LES WALE model was used. Due to the two-phase model for each time step more iterations (ten) were needed.

When cavitation was modeled the homogenious two-phase model was used and fluid density was calculated from the values of water density $\rho_{1}$ and water vapour density $\rho_{2}$ by the term $\rho=r_{1} \rho_{1}+r_{2} \rho_{2}$, where $r_{1}$ and $r_{2}$ are volume fractions of water and water vapour respectively and $r_{1}+r_{2}=1$. In regions without cavitation 


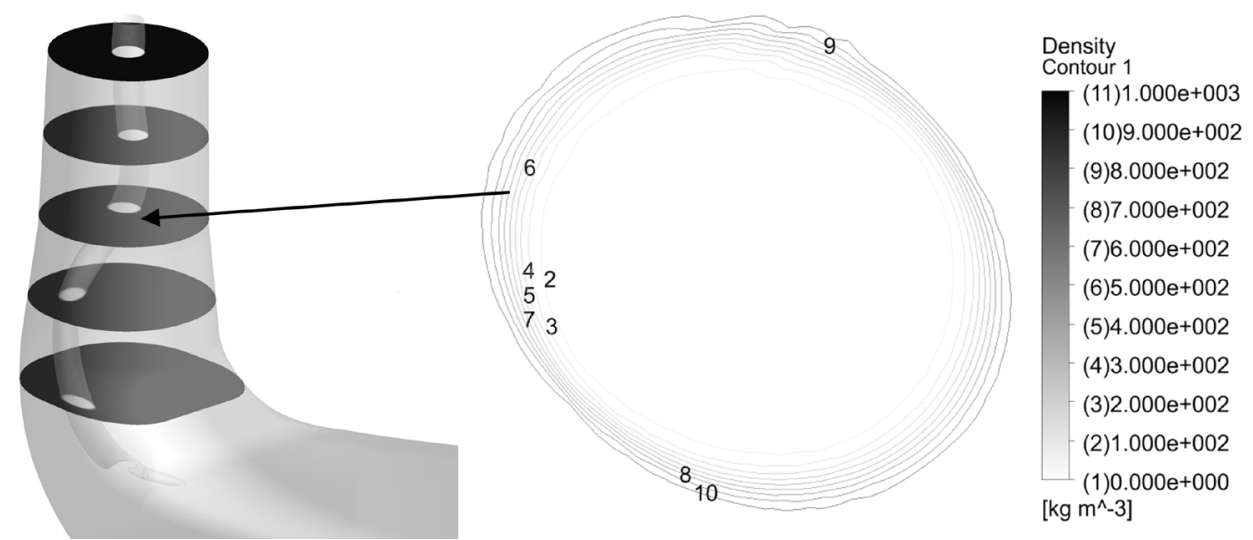

Fig. 8. Density at five horizontal cross-sections and a detail at one section, results of SAS-SST model with cavitation, case 2

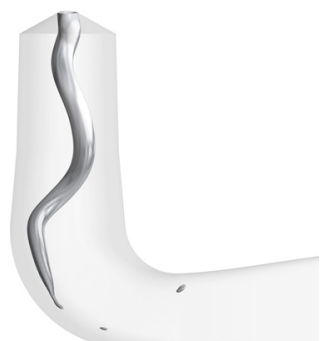

a)

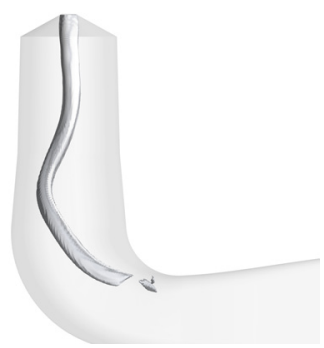

d)

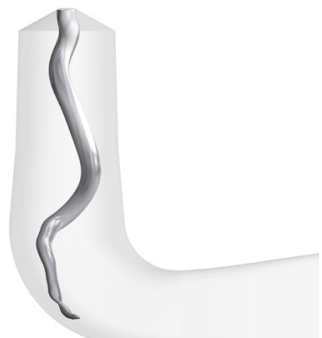

b)

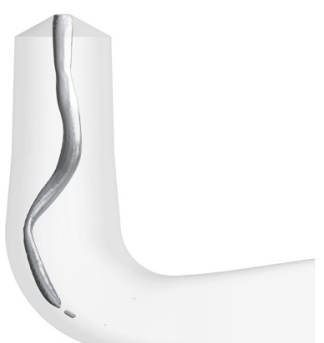

e)

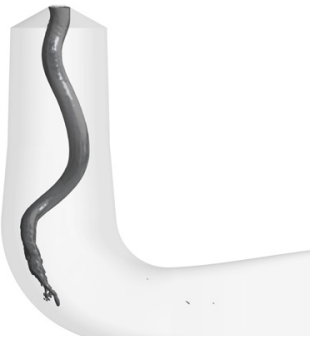

c)

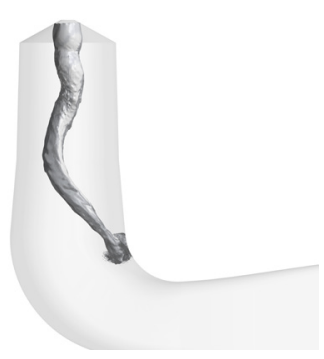

f)

Fig. 9. Numerically obtained shape of the vortex rope, case 2; a) SAS- SST, 20 runner revolutions, no cavitation, b) $\omega$-RSM, 20 runner revolutions, no cavitation, c) LES, 17 runner revolutions, no cavitation, d) SAS-SST, 20 runner revolutions, cavitation, e) BSL-RSM,20 runner revolutions, cavitation, f) LES, 20 runner revolutions, cavitation

density is equal to water density. The cavitating vortex rope consists of water and water vapour, therefore the value of density inside the rope is in range between $\rho_{1}$ and $\rho_{2}$. Density at five horizontal cross-sections is presented in Fig. 8. It can be seen that the core of the vortex rope mostly consists of water vapour and density there is close to $\rho_{2}$. The results in Fig. 8 were obtained by the SAS-SST model, but the pictures obtained by the other two turbulent models (RSM and LES) would be quite similar.

The numerically obtained vortex rope can be presented as an iso-surface of the appropriate value of absolute pressure and in case of cavitation also as an iso-surface of the appropriate value of water vapour volume fraction or fluid density. Furthermore, the images are almost equal. In this case the shape of the vortex rope is presented as 
an iso-surface of vaporization pressure. When cavitation was not included all three turbulent models predict a shape of the vortex rope similar to the one observed on the test rig (Figs. 9a, b and c). When cavitation was included the formation of the cavitating vortex rope was slower. In case of BSL-RSM model about 5 runner revolutions were needed to get the final length of the rope while in case of SAS-SST only after 15 runner revolutions the final length of the rope was obtained. The formation of cavitating rope by LES model was even slower. The vortex ropes obtained by the cavitation model and different turbulent models can be seen in Figs. 9d, e and f. They have slightly different shapes. The difference is significant just behind the runner where the ropes obtained by SAS-SST and BSL-RSM go nearly straight down. The rope obtained by LES WALE model is after 20 runner revolutions still not fully developed, but its shape is in better agreement with the experiment.

Numerically obtained pressure pulsations at two positions at the cone of the draft tube are presented in Fig. 10. In all the cases it took a long time before the frequency and amplitudes stabilized. Looking at first the curves obtained without cavitation, it can be seen that at the beginning the results of different turbulent models were quite different, but with time they were becoming more similar. The situation was worse when cavitation was modeled. By SAS-SST model the pressure pulsation frequency stabilized after 25 runner revolutions and it was nearly the same as without cavitation, but the amplitude at position 2 was much too small. By BSL-RSM only 32 runner revolutions were done. It seems that numerical errors were accumulating during the calculation and pressure oscillation was increasingly irregular. LES with the cavitation needed more than 20 runner revolutions before the oscillations stabilized. The frequency and amplitudes are slightly larger than those obtained without cavitation and are therefore, closer to experimental values. By LES besides pressure oscillation of high amplitudes also small oscillations of high frequency and small amplitudes were obtained.

In Table 4 experimental and numerical values of pressure pulsation frequency and amplitudes are presented. Frequencies of pressure pulsation were obtained by Fast Fourier Transform, Hanning's window was used. The calculated values of frequency are smaller than the experimental ones for all turbulent models with and without cavitation, except for LES WALE model with cavitation, where the calculated value is $1.4 \%$ larger than the measured one and where the best agreement between numerical and experimental values was obtained. The calculated values of amplitudes are smaller than the measured one, especially for position 2 , where the discrepancies obtained without cavitation are $31.26,30.83$ and $34.75 \%$ for SAS-SST, RSM and LES respectively. When cavitation was modeled only LES WALE model gave useful results, but the amplitudes are still much smaller than the measured ones. The grid in the draft tube for LES is more refined than for the other two turbulent models, therefore it is difficult to say, whether the results are better because of the more advanced turbulent model or because of grid refinement. The results by BSL-RSM and SAS-SST model with cavitation may be improved by grid refinement and double precision calculation.

Table 4. Numerical and experimental results for case 2

\begin{tabular}{|l|c|c|c|}
\hline & Frequency [Hz] & Position 1 App [\%] & Position 2 App [\%] \\
\hline Experiment & 3.50 & 2.77 & 4.67 \\
\hline SAS-SST, cavitation not modeled & 3.26 & 2.25 & 3.21 \\
\hline$\omega$-RSM, cavitation not modeled & 3.21 & 2.57 & 3.23 \\
\hline LES Smagorinsky, cavitation not modeled & 3.12 & 2.32 & 2.96 \\
\hline SAS-SST, cavitation modeled & 3.32 & 2.67 & 2.00 \\
\hline BSL-RSM, cavitation modeled & $\begin{array}{c}\text { Results too } \\
\text { irregular }\end{array}$ & $\begin{array}{c}\text { Results too } \\
\text { irregular }\end{array}$ & $\begin{array}{c}\text { Results too } \\
\text { irregular }\end{array}$ \\
\hline LES WALE, cavitation modeled & 3.55 & 2.43 & 3.20 \\
\hline
\end{tabular}


a) SAS-SST, position 1

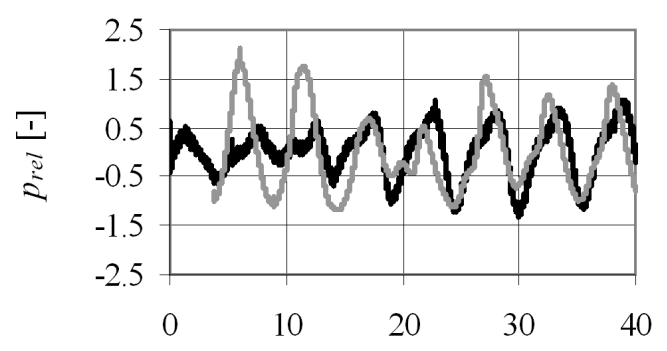

runner revolution [-]

c) RSM, position I

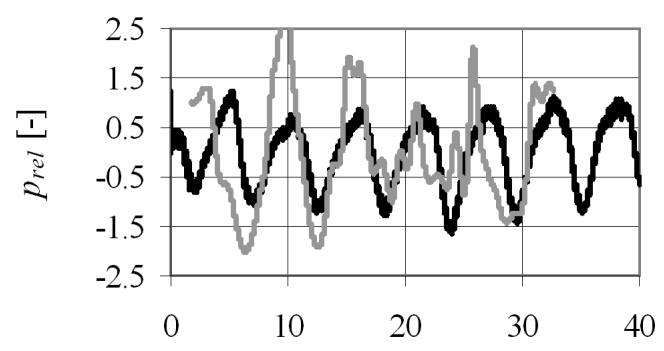

runner revolution [-]

e) LES, position 1

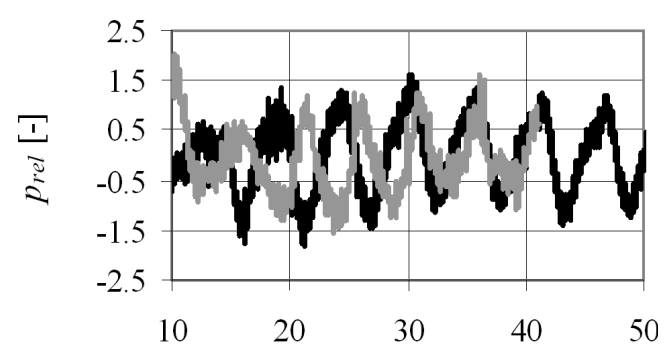

runner revolution [-]

cavitation not modeled, b) SAS-SST, position 2

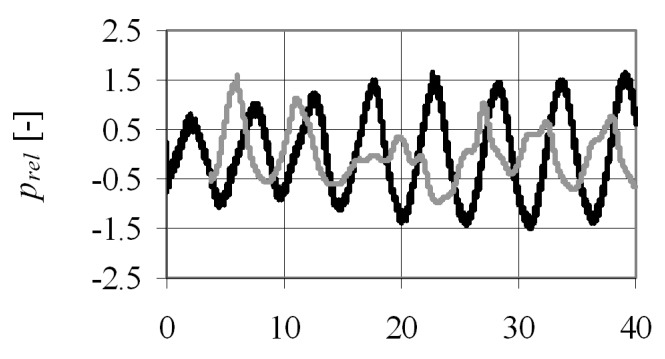

runner revolution [-]

d) RSM, position 2

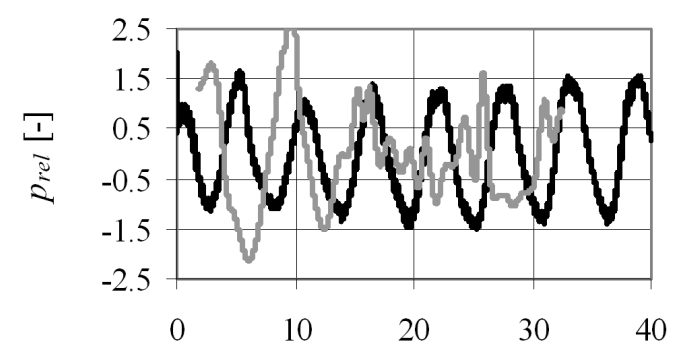

runner revolution [-]

f) LES, position 2

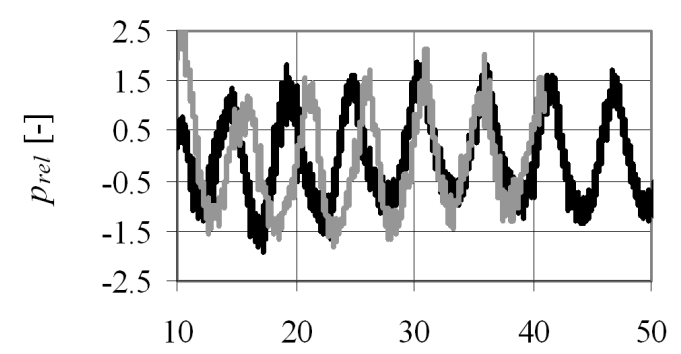

runner revolution [-]

cavitation modeled

Fig. 10. Pressure pulsation obtained by different turbulent models with and without cavitation modeling, case 2

\section{COMPUTATIONAL EFFORT AND \\ HARDWARE FOR VORTEX ROPE PREDICTION}

The numerical simulation of vortex rope is considerably time consuming. It takes a long time before the vortex acquires its final shape and the frequency of its rotation stabilizes. Usually more than 40 runner revolutions are needed to get stable values of pressure pulsation frequency and amplitudes. In case of RSM there are additional equations for Reynolds stresses, while for LES the 
grid has to be refined and time step reduced. The situation is even worse when cavitation is also modeled.

When the project Numerical simulation of vortex rope in a Francis turbine started, our computer capacities were limited to the cluster with 32 dual core processors. In May 2008 a supercomputer cluster with 2048 processor cores - 512 Quad-Core Intel Xeon processors L5335 was installed. In total the cluster has more than 2 TB RAM and for high performance computing communications InfiniBand is used. In case 1 the first mesh configuration (3.3 millions elements) was analyzed by the old computer and the CPU time for one complete runner revolution was about 24 hours on eight processors. For the second and third configurations of case 1 and for all analysis of case 2 the new supercomputer cluster was used. For the grid with 17 millions elements 32 quad-core processors (128 cores) were used and the total computational time was about ten hours for one runner revolution. For LES, where computational grid consists of about 23 millions elements, 64 quad-core processors (256 cores) were used and for one-phase flow two runner revolutions were performed per day. For the two phase flow with cavitation model more than one day was needed for one runner revolution. The usage of the higher number of processors for problems of about 20 million elements is not sensible because the reduction of the calculation time is too insignificant.

\section{CONCLUSION}

Nowadays powerful supercomputers enable the prediction of the rotating vortex rope in a Francis turbine. The results of case 1 showed that the experimentally and numerically obtained frequencies are very close, while the prediction of amplitudes is less accurate, but with grid refinement it approaches the experimental values. In case 2 it was shown that SAS-SST, RSM and LES models are suitable for vortex rope prediction and when cavitation was not modeled there was no significant difference in the accuracy of the results obtained by these three turbulent models. When cavitation was modeled the results obtained by SAS-SST and RSM were less accurate. However, we expect that they can be improved by grid refinement and double precision calculation. When LES model was used modeling of cavitation improved the results, amplitudes and especially frequency came closer to experimental values.

\section{ACKNOWLEDGEMENTS}

The research is partially financed by the Slovenian Research Agency ARRS - Contract no L2-1067-0263-08.

\section{NOMENCLATURE}

$A_{0} \quad[-]$

$A_{p p}[\%]$

$\begin{array}{ll}H & {[\mathrm{~m}]}\end{array}$

$H_{a t}[\mathrm{~m}]$

$H_{p} \quad[\mathrm{~m}]$

$H_{s}[\mathrm{~m}]$

$g \quad\left[\mathrm{~m} / \mathrm{s}^{2}\right]$

$p \quad[\mathrm{~Pa}]$

$p_{\text {aver }}[\mathrm{Pa}]$

$p_{\text {rel }}[-]$

$v \quad[\mathrm{~m} / \mathrm{s}]$

$\eta \quad[-]$

$\eta_{B E P}[-]$

$\sigma \quad[-]$

$\begin{array}{ll}\varphi & {[-]} \\ \rho & {\left[\mathrm{kg} / \mathrm{m}^{3}\right]} \\ \rho_{1} & {\left[\mathrm{~kg} / \mathrm{m}^{3}\right]} \\ \rho_{2} & {\left[\mathrm{~kg} / \mathrm{m}^{3}\right]} \\ \psi & {[-]}\end{array}$

BEP relative guide vane opening peak to peak pressure pulsation amplitude, $A_{p p}=\frac{\Delta p}{\rho g H} \cdot 100 \%$ head head corresponding to atmospheric pressure vaporization head suction head gravity pressure average pressure relative pressure, $p_{\text {rel }}=\frac{p-p_{\text {aver }}}{\rho g H}$ velocity efficiency at BEP cavitation coefficient, $\sigma=\frac{H_{a t}-H_{s}-H_{p}}{H}$ discharge coefficient density water density water vapour density pressure coefficient Best Efficiency Point efficiency

\section{REFERENCES}

[1] Kercan, V., Bajd, M., Djelić, V., Lipej, A., Jošt, D. (1995). Model and prototype draft tube pressure pulsations. Proceedings of the $7_{\text {th }}$ International Meeting of IAHR Work Group on the Behavior of Hydraulic 
Machinery under Steady Oscillatory Conditions, A6.

[2] Kercan, V., Bajd, M., Djelić, V., Lipej, A., Jošt, D. (1996). Model and prototype draft tube pressure pulsations. Proceedings of the XVIII. IAHR Symposyum on Hydraulic Machinery and Cavitation, Volume II, p. 994-1003.

[3] Kercan, V. (2001). The influence of Francis runner shape on phenomena of vortex rope in a draft tube. Ph.D thesis, University in Rijeka, Technical faculty. 94 p. (in Croatian)

[4] Skotak, A. (1999). Draft tube swirl modeling. Proceedings of the $9^{\text {th }}$ International Meeting of IAHR Work Group on the Behavior of Hydraulic Machinery under Steady Oscillatory Conditions, D4.

[5] Skotak, A. (2000). Of the helical vortex in the draft tube turbine modeling. $X X^{\text {th }} I A H R$ Symposium on Hydraulic Machinery and Systems.

[6] Ruprecht, A., Helmrich, T., Aschenbrenner, T., Scherer, T. (2002). Simulation of vortex rope in a turbine draft tube. Proceedings of the XXIst IAHR Symposium on Hydraulic Machinery and Systems.

[7] Skotak, A., Mikulašek, J., Lhotakova, L. (2002). Effect of the inflow conditions on the unsteady draft tube flow. Proceedings of the XXIst IAHR Symposium on Hydraulic Machinery and Systems.

[8] Sick, M., Doerfler, P., Sallaberger, M., Lohmberg, A., Casey, M. (2002). CFD simulation of the draft tube vortex. Proceedings of the XXIst IAHR Symposium on Hydraulic Machinery and Systems.

[9] Guo, Y., Kato, C., Miyagawa, K. (2006). Large-eddy simulation of non-cavitating and cavitating flows in an elbow draft tube. 23rd IAHR Symposium on Hydraulic Machinery and Systems.

[10] Kurosawa, S., Satou, S. (2006). Turbulent flow simulation for the draft tube of a Kaplan turbine. 23rd IAHR Symposium on Hydraulic Machinery and Systems.

[11] Miyagawa, K., Tsuji, K., Yahara, J., Nomura, Y. (2002). Flow instability in an elbow draft tube for a Francis pump-turbine. Proceedings of the XXIst IAHR Symposium on Hydraulic Machinery and Systems.

[12] Zhou, L, Wang, Z, Tian, Y. (2006). Numerical simulation of vortex cavitation in draft tube. 23rd IAHR Symposium on Hydraulic Machinery and Systems.

[13] Širok, B., Blagojević, B., Bajcar, T., Trenc, F. (2003). Simultaneous study of pressure pulsation and structural fluctuations of a cavitated vortex core in the draft tube of a Francis turbine. J. Hydraul. Res., vol. 41, no. 5, p. 541-548.

[14] Lipej, A., Jošt, D., Mežnar, V., Djelić, V. (2008). Numerical prediction of pressure pulsation amplitude for different operating regimes of Francis turbine draft tube. 24th IAHR Symposium on Hydraulic Machinery and Systems.

[15] Jošt, D., Lipej, A. (2009). Numerical prediction of the vortex rope in the draft tube. 3rd IAHR International Meeting of the Workgroup on Cavitation and Dynamic Problems in Hydraulic Machinery and Systems, p. 75-85.

[16] ANSYS CFX, Release 12, Solver Theory Documentation (2009). 